Check for updates

Cite this: RSC Adv., 2019, 9, 32137

Received 19th August 2019

Accepted 2nd October 2019

DOI: 10.1039/c9ra06476a

rsc.li/rsc-advances

\section{Water-soluble lanthanide coordination polymers particles with white-light emission and color tuning†}

\author{
Kaiqi Fan, (D) ${ }^{a}$ Xiaobo Wang, ${ }^{b}$ Yongpeng $\mathrm{Ma},{ }^{\mathrm{a}}$ Yu Li, ${ }^{\mathrm{a}}$ Guanglu Han, ${ }^{\text {a }}$ Zhigang Yin $^{\text {*a }}$ \\ and Jian Song*c
}

Water-soluble polymer particles (PPs) with strong fluorescence emission were prepared from hyperbranched poly(ethylenimine) (PEI) and terpyridine-bearing aldehyde (TPy) via Schiff base reaction and self-assembly in aqueous phase. TPy/PEI PPs were then used to develop a series of luminescent lanthanide coordination polymers particles (Ln-CPPS). The optical properties of these Ln-CPPs are readily modulated over a wide spectrum in water systems. Finally, water-soluble white-emitting Ln-CPPs were achieved by controlling the lanthanide ion stoichiometry. This Ln-CPPs design approach offers a robust pathway for white-luminescent materials in water systems.
In recent decades, dynamic metal coordination polymers (MCPs) have attracted great interest in catalysis, drug delivery, chemical sensors and bioanalysis applications. ${ }^{1-7}$ M-CPs are constructed from metal ions and organic ligands with a variety of structures and interesting properties for many potential applications. M-CPs acting as chemical sensors are mainly explored by making use of their luminescence properties..$^{8-10}$ The luminescent M-CPs can emit a stable and intense luminescent emission, so some substances can be detected by observing changes in luminescence intensity. It is well known that lanthanide ions have high color purity and long lifetime excitation lifetime, and the emission covers the entire visible range of 400 to $700 \mathrm{~nm}$. In particular, $\mathrm{Eu}$ (III) and $\mathrm{Tb}$ (III) ions can emit intense red and green light, respectively. Lanthanide coordination polymers (Ln-CPs) are promising luminescent materials because lanthanide ions have similar chemical properties and two or more lanthanide ions can be randomly distributed in coordination polymers with metal sites, which can modulate the color and brightness of the emission. ${ }^{11}$ For the above reasons, Ln-CPs have attracted the attention of many scientists and have been effectively used to design multiple color and white light emitting materials. For example, He and co-workers ${ }^{12}$ developed a new fluorophore that exhibits white light by combining an Eu(III) moiety (red emission) with an

${ }^{a}$ School of Material and Chemical Engineering, Zhengzhou University of Light Industry, Zhengzhou 450002, P. R. China

${ }^{b} J o u r n a l$ Editorial Department, Zhengzhou University of Light Industry, Zhengzhou 450002, P. R. China

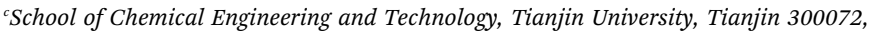
China.E-mail: songjian@tju.edu.cn

$\dagger$ Electronic supplementary information (ESI) available: Experimental section, synthesis and figures (S1). See DOI: 10.1039/c9ra06476a organic ligand (blue and green emission). Ma et al. ${ }^{13}$ reported a white-light-emitting $\mathrm{La}(\mathrm{III}) / \mathrm{Tb}$ (III)/Eu(III) coordination polymers based on combination of blue-emitting ligand/La(III), green-emitting $\mathrm{Tb}(\mathrm{III})$ and red-emitting Eu(III) units. Song et al. ${ }^{14}$ developed a white-light-emitting compound by doping a $\mathbf{E u ( I I I )}$ ion into the $\mathrm{Gd}(\mathrm{III})$ framework.

Meanwhile, the selection of suitable ligands plays a crucial role in the synthesis of Ln-CPs with good luminescent properties, since organic ligands can be used not only as building blocks for the construction of new backbones of Ln-CPs, but also as effective sensitizer for Ln(III) ions. ${ }^{15,16}$ However, ligands are generally poorly water soluble, which limits the practical sensing application in environmental and biological systems. ${ }^{17}$ An efficient strategy to promote dispersion in water is to prepare Lanthanide coordination polymer particles (Ln-CPPs) by miniemulsion method, reprecipitation method, and so on..$^{18}$ Nevertheless, several drawbacks still exist for their preparation and application, such as sophisticated multistep synthetic pathways, use of environmentally unfriendly organic solvents, and the possibility of fluorescence self-quenching in aqueous solution. Therefore, the systematic investigation of water-soluble Ln-CPPs with white-light emission is quite rare. More research studies are urgently needed to accelerate the development of white-light luminescent Ln-CPPs in the water system.

Based on the above considerations, we rationally designed water-soluble polymer particles with blue emission and selected $\mathrm{Tb}(\mathrm{III} / \mathrm{Eu}$ (III) to construct white-light-emitting Ln-CPPs (Fig. 1). The water-soluble polymer particles were constructed from hyperbranched poly(ethylenimine) (PEI) and terpyridinebearing aldehyde (TPy) via Schiff base reaction and selfassembly. Structural characterization and luminescence properties in the water system of Ln-CPPs are studied in detail. An 


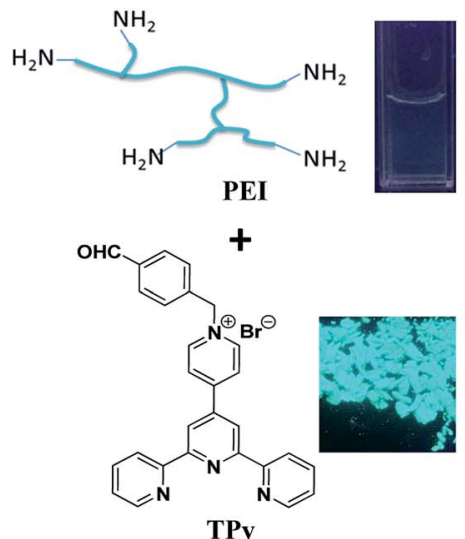

TPy
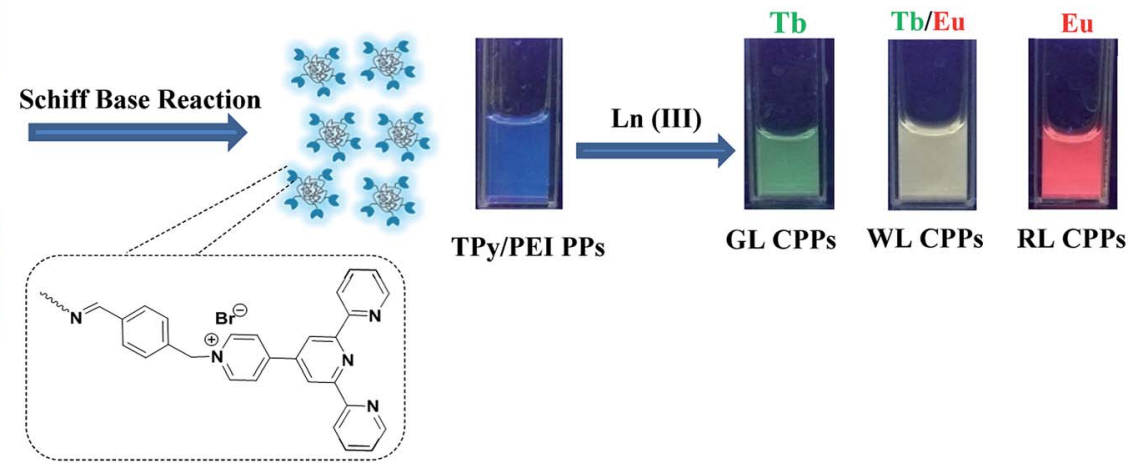

Fig. 1 Schematic preparation of TPy/PEI PPs and Ln(III) coordination-based luminescent polymer particles (Ln-CPPs) under UV light ( $\lambda_{\text {ex }}=365$ $\mathrm{nm})$.

important clue could be obtained from the result that Ln-CPPs constructed by terpyridine ligands can maintain their structural and luminescent properties in the water system. This research also provides a basis for realizing the controllability of watersoluble white-light-emitting Ln-CPPs in the future.

Synthesis of the TPy/PEI PPs is based on facile Schiff base reaction, which refers to the reaction between primary amine on PEI and aldehyde group on TPy, resulting in a product containing $\mathrm{C}=\mathrm{N}$ bonds. Moreover, the diluted TPy/PEI PPs solution emits blue fluorescence under a $365 \mathrm{~nm}$ UV lamp. Fig. 2A displays the fluorescence excitation and emission spectra of the TPy/PEI PPs solution, and the maximum excitation and emission wavelengths are 330 and $448 \mathrm{~nm}$, respectively. The UV-vis absorption spectra of TPy/PEI PPs, PEI and TPy were shown in Fig. S4. $\dagger$ Compared with TPy, the absorption peak at $250 \mathrm{~nm}$ in TPy/PEI PPs solution is weakened, which may be due to the decolorization effect caused by the formation of copolymer by TPy and PEI. In addition, the absorption peak at $335 \mathrm{~nm}$ in TPy/ PEI PPs solution is attributed to $\mathrm{n} \rightarrow \pi^{*}$ transitions of $\mathrm{C}=\mathrm{N}$ bonds. ${ }^{18,19}$ These phenomena indicated TPy/PEI PPs were a newly generated subject.

The morphologies of TPy/PEI PPs were characterized by transmission electron microscopy (TEM), Fig. S5A $\dagger$ is a TEM image and reveals that the TPy/PEI PPs are monodisperse spherical shape with the size distribution in the range of 26$50 \mathrm{~nm}$. Formation of water-soluble nanoparticles is due to the following factors. In TPy/PEI copolymer, ample amine groups and pyridinium groups are hydrophilic, whereas Schiff base bonds are hydrophobic. As a result, the hyperbranched structure of TPy/PEI copolymer tends to fold and collapse, shrinking and self-assembling into uniform polymer nanoparticles in aqueous medium. ${ }^{18}$ Many hydrophilic groups on the surface of TPy/PEI PPs make the excellent water dispersity possible. To further explore the chemical composition of TPy/PEI PPs, we performed FT-IR spectra of PEI, TPy, and TPy/PEI PPs (Fig. 2B). Several featured vibration bands at 3284 and $1590 \mathrm{~cm}^{-1}$ in PEI are associated with the stretching vibration of $\mathrm{N}-\mathrm{H}$ bond, and their intensity is decreased in TPy/PEI PPs, which indicates that some amine groups have reacted with TPy. In addition, another remarkable new peak at $1630 \mathrm{~cm}^{-1}$ was observed in TPy/PEI PPs, which can be assigned to the $\mathrm{C}=\mathrm{N}$ bond. ${ }^{20-25}$ Meanwhile, a new peak at $8.37 \mathrm{ppm}$ was observed in the ${ }^{1} \mathrm{H}$ NMR spectra of TPy/PEI PPs (Fig. S6 $\dagger$ ), which can be assigned to $\mathrm{N}=\mathrm{CH}$ protons. ${ }^{26}$ The monitoring of the aldehyde conversion into imine units can be carried out by measuring the $\mathrm{C} \underline{\mathrm{HO}} / \mathrm{C} \underline{\mathrm{H}}=\mathrm{N}$ integral ratio, and the conversion rate of the aldehyde into
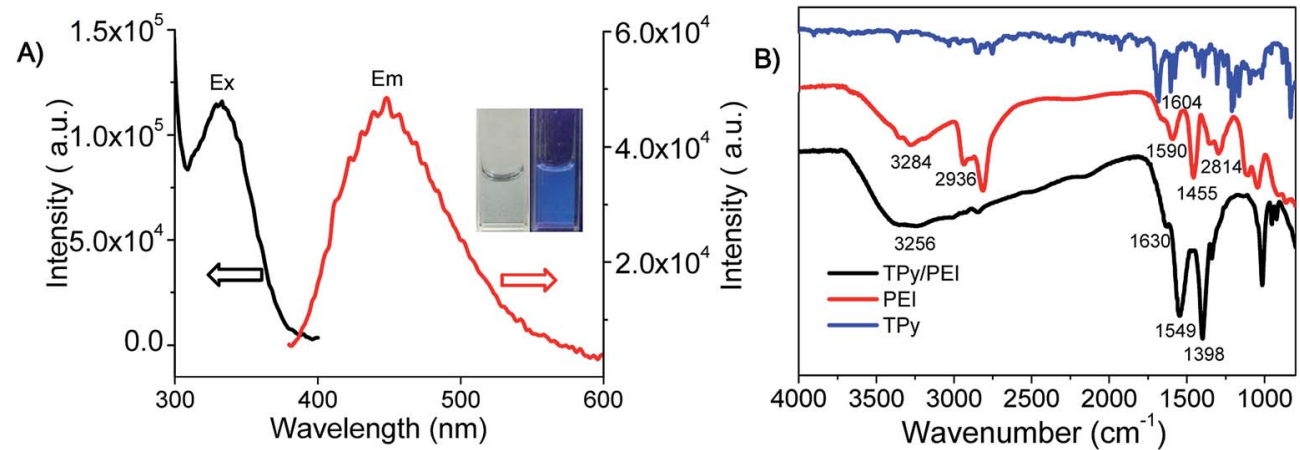

Fig. 2 (A) Fluorescence excitation and emission spectra of TPy/PEI PPs $\left(0.01 \mathrm{~g} \mathrm{~mL}^{-1}\right)$. (Inset) Photographs of TPy/PEI PPs under visible light and UV light of $365 \mathrm{~nm}$. (B) FT-IR spectra of TPy/PEI, PEI, and TPy. 


\section{A)}
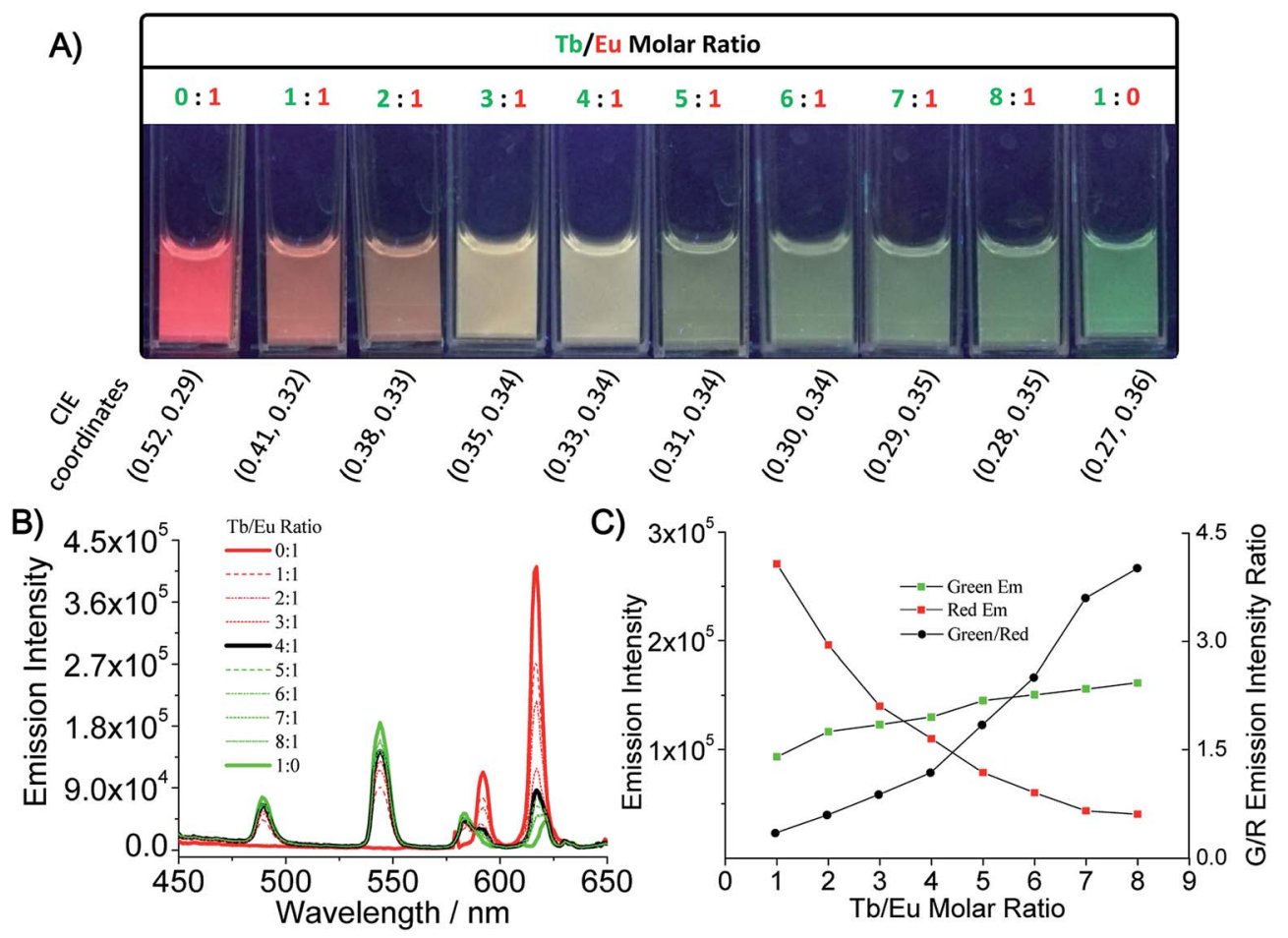

Fig. 3 Luminescence tuning: (A) photographs of Ln-CPPs under UV irradiation, corresponding CIE coordinates are mentioned below; (B) emission spectra ( $\lambda_{\text {ex }}=330 \mathrm{~nm}$ ) of Ln-CPPs and (C) Job's plot showing the peak emission intensity of the red band at $544 \mathrm{~nm}$ and green band at $616 \mathrm{~nm}$ as a function of the Tb/Eu molar ratio $(1: 1,2: 1,3: 1,4: 1,5: 1,6: 1,7: 1$, and $8: 1)$.

imine units is $69 \%$. The estimation of the conversion rate from the ${ }^{1} \mathrm{H}$-NMR spectrum agrees well with the calculation from the weighting measurements with a conversion rate of $73 \%$. These analysis results well demonstrated the formation of Schiff base bonds between TPy and PEI.

TPy/PEI PPs possess intrinsic fluorescence, good water solubility, and functional terpyridine structure unit, allowing us to incorporate the $\mathrm{Ln}$ (III)-TPy coordination complexes into polymer networks. With the incremental addition of $\mathrm{Tb}\left(\mathrm{NO}_{3}\right)_{3}$ to the TPy/PEI PPs solution $(2 \% \mathrm{v} / \mathrm{v})$, the TPy : Ln ratio is $2: 1$, which produces green-luminescent Ln-CPPs, GL CPPs $(\tau=0.35$ $\mathrm{ms}, \Phi=4.3 \%$, CIE coordinates $(0.27,0.36)$, Fig. S7, S8 and Table $\mathrm{S} 1 \dagger)$. In the corresponding emission spectrum, a decrease in the luminescence intensity of the ligand centred emission band at $448 \mathrm{~nm}$ with the concomitant emergence of sharp bands at $489 \mathrm{~nm}, 544 \mathrm{~nm}, 583 \mathrm{~nm}$, and $622 \mathrm{~nm}$ was observed (Fig. S7†). A decrease in the luminescence intensity of the central emission band of the ligand was observed. These emission bands were assigned to ${ }^{5} \mathrm{D}_{4}{ }^{-7} \mathrm{~F}_{6},{ }^{5} \mathrm{D}_{4}-^{7} \mathrm{~F}_{5},{ }^{5} \mathrm{D}_{4}{ }^{-7} \mathrm{~F}_{4}$, and ${ }^{5} \mathrm{D}_{4}{ }^{-7} \mathrm{~F}_{3}$ based transitions, respectively, for $\mathrm{Tb}(\mathrm{III}){ }^{27-30} \mathrm{~A}$ similar procedure was observed upon addition of $\mathrm{Eu}\left(\mathrm{NO}_{3}\right)_{3}$ to the TPy/PEI PPs solution with the occurrence of five characteristic Eu(III)-based emission bands having maxima at $579 \mathrm{~nm}\left({ }^{5} \mathrm{D}_{0}{ }^{7} \mathrm{~F}_{0}\right), 592 \mathrm{~nm}\left({ }^{5} \mathrm{D}_{0}{ }^{-} \mathrm{F}_{1}\right)$, $617 \mathrm{~nm}\left({ }^{5} \mathrm{D}_{0}{ }^{7} \mathrm{~F}_{2}\right), 649 \mathrm{~nm}\left({ }^{5} \mathrm{D}_{0}{ }^{7} \mathrm{~F}_{3}\right)$, and $687 \mathrm{~nm}\left({ }^{5} \mathrm{D}_{0}{ }^{-}{ }^{7} \mathrm{~F}_{4}\right)$, resulting in a clear red-luminescent Ln-CPPs, RL CPPs $(\tau=0.81$ $\mathrm{ms}, \Phi=11.3 \%$, CIE coordinates $(0.52,0.29))$. These emission spectra demonstrate that $\mathrm{Ln}^{3+}\left(\mathrm{Eu}^{3+}\right.$ or $\left.\mathrm{Tb}^{3+}\right)$ ions were successfully doped to the TPy/PEI PPs. More importantly, strong fluorescence could still be detected even after these Ln-CPPs were stored for over a week, implying that the coordination between the TPy/PEI PPs and $\mathrm{Ln}^{3+}$ ions is very stable. The interactions between the TPy/PEI PPs and $\mathrm{Ln}^{3+}$ ions were further monitored by FT-IR spectroscopy (Fig. S9†). Strong absorbent bands at 3256, 1549 and $1398 \mathrm{~cm}^{-1}$ in TPy/PEI PPs are attributed to the stretching vibrations of $\mathrm{N}-\mathrm{H}$ bond. ${ }^{18,19}$ After the formation of RL CPPs, GL CPPs or WL CPPs using $\mathrm{Ln}^{3+}$ ions, a dramatically red shift appeared, which indicated the coordination of the TPy/PEI PPs to $\mathrm{Ln}^{3+}$ ions. The medium-to-weak bands at $760 \mathrm{~cm}^{-1}$ for RL CPPs, $769 \mathrm{~cm}^{-1}$ for GL CPPs and $765 \mathrm{~cm}^{-1}$ for WL CPPs are observed as additional evidence of the $\mathrm{Ln}-\mathrm{N}$ formation. ${ }^{31}$

Next we investigated how to modulate the emission of polymer particles by adjusting the stoichiometry of the two lanthanide chromophores. Titration of the $\mathrm{Tb} / \mathrm{Eu}$ molar ratio resulted in a series of Ln-CPPs with a broad spectrum of emission under UV irradiation (Fig. 3A). By testing the emission spectrum (Fig. 3B and C), it was found that the intensity of the green band at $544 \mathrm{~nm}$ increased gradually at the expense of the intensity of the red band at $616 \mathrm{~nm}$ as a function of $\mathrm{Tb} / \mathrm{Eu}$ molar ratio. Interestingly, an intense white-luminescent Ln-CPPs, WL CPPs (CIE coordinates $(0.33,0.34)$ ), were observed when the Eu/ $\mathrm{Tb}$ molar ratio was $1: 4$. The smart illumination control strategy here provides a simple design approach for broad-spectrum color adjustment of luminescent polymer materials.

In conclusion, we created polymer particles with blue emission from PEI and TPy via Schiff base reaction and self-assembly 
under mild conditions. The structural characterization and the fundamental properties of the TPy/PEI PPs have been studied. Because of the specific structure, the TPy/PEI PPs exhibit excellent water solubility. Furthermore, we have used the TPy/ PEI PPs to develop a series of luminescent Ln-CPPs with $\mathrm{Eu}(\mathrm{III}), \mathrm{Tb}$ (III), and mixed $\mathrm{Eu}(\mathrm{III}) / \mathrm{Tb}$ (III) in aqueous medium. The individual Ln-CPPs exhibited bright red (Eu-CPPs) and green (Tb-CPPs) fluorescence upon exposure to UV light $\left(\lambda_{\mathrm{ex}}=365\right.$ $\mathrm{nm}$ ). Careful tuning of the stoichiometric ratio of $\mathrm{Eu}(\mathrm{III})$ and $\mathrm{Tb}$ (III) helped in achieving water-soluble white-emitting LnCPPs, which could offer a suitable pathway for preparing white-luminescent materials in water systems. Due to their stability in water, in our next work efforts will be focused on exploring their potential applications in biological and environmental areas as luminescence sensing and quantitative detection materials.

\section{Conflicts of interest}

There are no conflicts to declare.

\section{Acknowledgements}

This research was supported by the National Natural Science Foundation of China (No. 21671178) and Scientific Research Foundation for the Doctoral Program (No. 2014BSJJ061).

\section{Notes and references}

1 A. Y.-Y. Tam and V. W.-W. Yam, Chem. Soc. Rev., 2013, 42, 1540.

2 X. Yu, L. Chen, M. Zhang and T. Yi, Chem. Soc. Rev., 2014, 43, 5346.

3 G. L. Fiore, S. J. Rowan and C. Weder, Chem. Soc. Rev., 2013, 42, 7278.

4 C. Neaime, C. Daiguebonne, G. Calvez, S. Freslon, K. Bernot, F. Grasset, S. Cordier and O. Guillou, Chem. - Eur. J., 2015, 21, 17466.

5 K. Fan, Z. Su, S. Wang, S. Zhao, G. Han, S. Yu, B. Zhang, Z. Yin and J. Song, J. Zhengzhou Univ. Light Ind., Nat. Sci. Ed., 2018, 33, 56.

6 K. Fan, H. Kong, X. Wang, X. Yang and J. Song, RSC Adv., 2016, 6, 80934.

7 J. Liu, L. Chen, H. Cui, J. Zhang, L. Zhang and C.-Y. Su, Chem. Soc. Rev., 2014, 43, 6011.
8 K. Fan, J. Yang, X. Wang and J. Song, Soft Matter, 2014, 10, 8370.

9 B. Y. Guan, A. Kushima, L. Yu, S. Li, J. Li and X. W. Lou, Adv. Mater., 2017, 29, 1605902.

10 K. Fan, X. Wang, Z. Yin, C. Jia, B. Zhang, L. Zhou and J. Song, J. Mater. Chem. C, 2018, 6, 10192.

11 J. Jia, J. Xu, S. Wang, P. Wang, L. Gao, M. Yu, Y. Fan and L. Wang, CrystEngComm, 2015, 17, 6030.

12 G. He, D. Guo, C. He, X. Zhang, X. Zhao and C. Duan, Angew. Chem., Int. Ed., 2009, 48, 6132.

13 M.-L. Ma, C. Ji and S.-Q. Zang, Dalton Trans., 2013, 42, 10579.

14 S. Song, X. Li and Y.-H. Zhang, Dalton Trans., 2013, 42, 10409.

15 M. Reddy and S. Sivakumar, Dalton Trans., 2013, 42, 2663.

16 N. M. Shavaleev, S. V. Eliseeva, R. Scopelliti and J.-C. G. Bünzli, Inorg. Chem., 2010, 49, 3927.

17 Y. Liu, J. Ma, C. Xu, Y. Yang, M. Xia, H. Jiang and W. Liu, Dalton Trans., 2018, 47, 13543.

18 S. G. Liu, D. Luo, N. Li, W. Zhang, J. L. Lei, N. B. Li and H. Q. Luo, ACS Appl. Mater. Interfaces, 2016, 8, 21700.

19 J. Li, B. Li and M. Liu, Sens. Actuators, B, 2019, 280, 171.

20 S. G. Liu, N. Li, Y. Ling, B. H. Kang, S. Geng, N. B. Li and H. Q. Luo, Langmuir, 2016, 32, 1881.

21 D. Wang and T. Imae, J. Am. Chem. Soc., 2004, 126, 13204.

22 W. I. Lee, Y. Bae and A. J. Bard, J. Am. Chem. Soc., 2004, 126, 8358.

23 L. Pastor-Pérez, Y. Chen, Z. Shen, A. Lahoz and S. E. Stiriba, Macromol. Rapid Commun., 2007, 28, 1404.

24 L. Guo, S. Wu, F. Zeng and J. Zhao, Eur. Polym. J., 2006, 42, 1670.

25 I. Lee, S. Kim, S.-n. Kim, Y. Jang and J. Jang, ACS Appl. Mater. Interfaces, 2014, 6, 17151.

26 S. A. Lee, G. R. You, Y. W. Choi, H. Y. Jo, A. R. Kim, I. Noh, S.-J. Kim, Y. Kim and C. Kim, Dalton Trans., 2014, 43, 6650.

27 M. C. Heffern, L. M. Matosziuk and T. J. Meade, Chem. Rev., 2013, 114, 4496.

28 H. Agarwalla, K. Jana, A. Maity, M. K. Kesharwani, B. Ganguly and A. Das, J. Phys. Chem. A, 2014, 118, 2656.

29 T. S. Mahapatra, S. Chaudhury, S. Dasgupta, V. Bertolasi and D. Ray, New J. Chem., 2016, 40, 2268.

30 T. S. Mahapatra, H. Singh, A. Maity, A. Dey, S. K. Pramanik, E. Suresh and A. Das, J. Mater. Chem. C, 2018, 6, 9756.

31 J. Pei, X. L. Liu, W. L. Yu, Y. H. Lai, Y. H. Niu and Y. Cao, Macromolecules, 2002, 35, 7274. 